\title{
A Feasible Interpretation of the Wave-particle Duality Origin of
}

\author{
Microscopic Matter Particles
}

\author{
Wei Fan \\ Yunnan Institute of Modern Physics Kunming 65000 China
}

\begin{abstract}
:
Classical mechanics describes the laws of motion in the macroscopic material world, while quantum mechanics describes the laws of motion in the microscopic material world that classical mechanics cannot explain, and achieves a highly accurate mathematical representation of the laws of microscopic physical motion. But even with such a successful theory, there is confusion about the probability wave. Therefore, this paper attempts to improve the physical definition of the conceptual basis of quantum mechanics, thus solving the confusion of quantum mechanical probability waves, and finally proposes a feasible interpretation of the wave-particle duality origin of microscopic matter particles.
\end{abstract}

Keywords: Quantum mechanics; Wave-particle duality; Quantum entanglement; Cosmic redshift;

\section{1: Introduction}

The question of the volatility and particle-like nature of light is one that has been going on since Newton's time. It was not until the establishment of quantum mechanics in the last century that we realized the wave-particle duality of light and other microscopic particles. However, what is the origin of wave-particle duality? How to interpret the confusion of quantum mechanical probability waves? What is the origin of quantum and quantum entanglement? Through the existing quantum theory, we still can't get a good explanation, so this paper tries to perfect the existing quantum mechanics theory to solve these problems. At the same time, I read the relevant literature and learned that many people try to solve these problems $[1,2,3,4,5,6,7,8,9,10,11,12,13,14,15,16]$, but I will use a different way to explain these problems.

In classical mechanics, Newtonian mechanics and geometric optics belong to the physics theory that studies the laws of macroscopic material world movement, and the research object is macroscopic individual; Huygens' wave optics is a physics theory that studies the laws of group motion in the macroscopic material world, and the research object is a macro group. By the end of the 19th century, it was discovered that the old classical theory could not explain the microscopic material system, so quantum mechanics was founded in the early 20th century. Quantum mechanics is a set of theories that study the laws of motion of microscopic material systems. The research object is individuals and groups in the microscopic material system, thus making up for the shortcomings of classical mechanics at the micro level.
However, the existing theory of quantum mechanics is not a perfect theory, it has many pain points, so this article attempts to solve these pain points.

By thinking, we can find that the law of microscopic particle motion in the material world is a group phenomenon rather than an individual phenomenon; due to the influence of Newtonian mechanics, when our scientists try to describe the laws of motion of microscopic material systems, they always habitually study the group behavior of microscopic material systems from the perspective of individuals, thus trying to find the position and momentum of the particles in the microscopic material system, which leads to the confusion of quantum mechanical probability waves. In fact, our scientists do not know, for the motion law of a group of particles, we are only suitable to study the energy density of this group of particles and not suitable for studying the position and momentum of this group of particles. Therefore, when we switch the individual perspective of traditional quantum mechanics to the group perspective, we solve the confusion of quantum mechanical probability waves.

In addition, we also found that the key reason for the confusion of quantum mechanics in generating probability waves is that Copenhagen Interpretation only uses one kind of mathematics (probability) to interpret another kind of mathematics (wave function). Because it leads a physical problem to the mathematical level for research, it leads us to think that the mathematical probability is the physical reality, which leads to the confusion of the quantum mechanical probability 
wave. In fact, the Copenhagen Interpretation does not give the wave function a real physical meaning. Therefore, this paper attempts to give the wave function a real physical interpretation, and proposes a feasible interpretation of the origin of the wave-particle duality of microscopic particles.

At the same time, based on the final interpretation of the essence of light at the micro level, this paper also proposes another feasibility interpretation method about the principle of the redshift of the universe and the constant speed of light.

\section{2: A new interpretation of the wave-particle duality of light}

According to quantum optics [17], electrons emit photons and periodically move around the nucleus in unfixed orbits. Because electrons do not produce losses after the photons are emitted, electrons will not be able to transfer energy outwards without the presence of a medium. So, we can put forward a kind of conjecture, the light can be a medium wave. Thus, the frequency $v$ of the light wave is equal to the number of times the electron is wound around the core per unit time $n$ :

$$
v=n=\frac{1}{T}
$$

For an electronic through outward transfer the kinetic energy of the collision in the medium, can be defined as a constant, we here called the Planck's constant, because the electrons are doing around the nuclear periodic motion, so there is a time interval between cycle, then the radiation energy, it is not continuous, and can only be one separate energy, so the light energy are:

$$
E=h n=h v
$$

As can be seen from the above, the origin of the photons is electronic cycle operation of a frequency of light waves of energy, that is, the origin of the quantum is a single wave has kinetic energy.

The photoelectric effect of current on the energy of electrons absorbing photons is as follows:

$$
h v=E_{k}+w
$$

Can be seen from the above, the electron around the nucleus cycle movement will inevitably lead to the radiation and the energy absorption is one of continuous, so we can be explained from the Angle of the volatility of light particles of light, and then explains the light quantum and wave-particle duality of light and origins.

Discussion: in fact, not only is the energy of light waves not continuous, but the energy of any wave is not continuous. Because any waves is a periodic vibration, there any vibration cycle time interval, the interval lead to the existence of the peaks and troughs, the discontinuity of the existence of the peaks and troughs caused energy, therefore, any wave of energy are continuous, we can go to the measurement of sound waves, sound waves have peaks and troughs, acoustic energy must also be continuous).In other words, the discontinuity of energy is an intrinsic property of waves, not unique to light waves.

Considering that the energy of electron radiation fluctuates at a macro level, it satisfies the huygens fresnel principle:

$$
d E=\frac{K d s}{L} \cos \left(\omega t-\frac{2 \pi L}{\lambda}\right)
$$

Vibration to:

$$
E=\int d E=\int_{S} \frac{K d s}{L} \cos \left(\omega t-\frac{2 \pi L}{\lambda}\right)
$$

Considering the light waves can be spread in vacuum, liquid, gas, solid, suggesting that light was a medium instead of shear waves (later), micro mathematical expression can be represented as:

$$
\tau_{j i, j}+\rho f_{i}=\rho \frac{\partial^{2} u_{i}}{\partial t^{2}}
$$

The longitudinal wave velocity is:

$$
V=\sqrt{\frac{E}{\rho}}
$$

Because we think of light waves here as longitudinal waves rather than horizontal waves, we can only use the mathematical expression of longitudinal waves.

\section{The explanation is as follows:}

For polarization phenomena of the shear properties of light waves, we can be interpreted as: as the light of the longitudinal wave through the solid medium (polaroid), caused by the secondary vibration (solid polaroid into new waves), because the polaroid as displacement of solid medium is not going to happen, will happen only shear deformation, so the light waves from compressional to shear wave (such as sound waves travel through solid when converting from longitudinal shear wave), thus, light is the nature of the shear wave. So, we can use the p-wave explained marius polarization is found (note: we think that light is a transverse wave, because marius discovered the phenomenon of the polarization of the light, and marius polarization phenomenon is found before, people all agree that 
light is longitudinal wave).

Discussion: the traditional theory that light waves are shear waves is actually a misunderstanding. Because shear waves require solid media, and the existence of solid media will cause stars to be unable to move, and astronomical observations show that all stars are moving, so light waves cannot be shear waves. Because light wave is the energy of electron vibration radiation, electron vibration belongs to mechanical motion, so light wave belongs to mechanical wave. (note: Light is a mechanical wave based on 20th century quantum optics [17]. And Marius's law is based on the 19th century, so I don't know that light waves are mechanical waves.).

At the same time, it can be seen from the above equation that the speed of light is inversely proportional to the density of the medium, which is expressed as:

$$
V \propto \frac{1}{\rho}
$$

According to Maxwell's electromagnetic theory, the wave velocity of electromagnetic waves is inversely proportional to the relative capacitance and relative magnetic conductivity of the medium:

$$
V \propto \frac{1}{\boldsymbol{\varepsilon}_{r} \boldsymbol{U}_{r}}
$$

Considering that light wave is a kind of electromagnetic wave and light wave is a medium wave, electromagnetic wave is also a medium wave, so the relative capacitance of the medium is positively proportional to the relative magnetic conductivity and the density of the medium.

$$
\varepsilon_{r} u_{r} \propto \rho
$$

The wave velocity of electromagnetic waves is inversely proportional to the density of the medium:

$$
V \propto \frac{1}{\boldsymbol{\varepsilon}_{r} \boldsymbol{U}_{r}} \propto \frac{1}{\rho}
$$

Considering the medium p-wave alone, under the flat space-time, relative to the stars of the gravitational field distribution, so must light medium density distribution under the effect of gravity, which light is a kind of medium density wave. So, medium density is proportional to the gravitational field intensity distribution, then medium density is proportional to the potential gradient of gravity negative:

$$
\rho \propto E=-\nabla \gamma P
$$

Among them, is an undetermined constant. Can be seen from the type of light medium density and field intensity proportional relations, due to the speed of light and medium density is inversely proportional relationship, so the speed of light and gravitational field intensity is inversely proportional relationship is expressed as:

$$
V \times E=k(k \neq 0)
$$

Where, it's a constant. $k$ Can be seen from the above, the strength of the gravitational field is strong, the slower the speed of light, presents gradient distribution in the speed of light in the gravitational field at the same time. So, we can by observing the same at different positions of field strength of the gravitational field of the speed of light and gravitational field intensity near the speed of light value to the size of the experiment.

Due to the strong gravitational field of the light medium density is relatively large, so, when a beam of light through the strong gravitational field, belongs to the hydrophobic medium to light dense medium transmission. Thus, when a beam of light passes through has strong gravitational field near objects, the light will inevitably occur obvious refraction phenomenon, of course the bending phenomenon have been confirmed by astronomical observations, and the deflection rate satisfies the refractive index's law:

$$
n=\frac{c}{v}
$$

Since the speed of light in the same speed is not a fixed value in gravitational field, but a change of gradient value, so the same gravitational field of the refractive index of light should be a change with strong gravitational field gradient range value, meet the relationship:

$$
n \propto \rho \propto E=-\nabla \gamma P
$$

With the actual astronomical observations of light deflection is a range of values. 
Considering the following relationship between wavelength and refractive index:

$$
\lambda^{\prime}=\frac{\lambda}{n}
$$

Among them, said a vacuum wavelength, said the vacuum wavelength of medium, the refractive index. Thus, we can see that when a beam of light through the strong gravitational field near, because of its refractive index change, whose wavelength would be smaller. When a beam of light emitted from the interior of the gravitational field, belongs to the dense medium to light thin medium propagation process, the refractive index decreases, then the wavelength will be larger, can produce red shift, this can explain the phenomenon of gravitational redshift.

\section{Discussion and prediction:}

A: relative to the stars by light transmission medium of the gravitational field distribution, then the gravitational field must be synchronized with the periodic movement of medium, we can through astronomical observations the existence of periodic motion of the medium disturbance wave, thus to prove whether light has the existence of the medium, to verify the rationality of the theory in this paper.

B: if there is medium, light and gravitational field relative to the stationary distribution. As we know, the earth on a certain object exploded, produces the high density of medium wave, the shock waves of light medium density is larger, we can make a beam of light through the shock wave, observing the speed of light is slow to prove the existence of light is a longitudinal wave is medium and light density medium.

\section{3: A new interpretation of the wave-particle duality of other matter particles}

It can be seen from the above that light is a wave of medium particles, and the light intensity represents the energy flow density at a certain point. The mathematical expression is:

$$
I=\bar{S}=\bar{\Omega} u=2 \pi^{2} \rho A^{2} v^{2} u
$$

So, from microscopic to discuss the position and momentum of the light has no physical meaning, can only discuss the space distribution of each point of energy flow density conform to the classical principle of wave optics, so there is no probability distribution problem. Thus, we can eliminate the confusion of light quantum probability distribution. Fluctuating when electrons pass through certain crystals. Because electronic is a periodic motion, said its wavelength is not electronic is a wave, said electronic cycle in the process of operation, a week before the movement of the electrons and a week after the movement of the electron orbital distance (or do translational motion of the spacing between the two group of electronic), satisfy the relationship:

$$
\lambda=\frac{2 \pi r}{n}
$$

Where the frequency represents the number of times the electron moves around the nucleus per unit time (or the number of times the electron passes through a point in space per unit time).

$$
v=n=\frac{2 \pi r}{\lambda}
$$

So, we think, electronic or other microscopic particles (such as neutron, atoms, etc.) through certain crystal wave diffraction phenomenon happens, said is not the wave is a kind of microscopic particle, but the elastic wave scattering through the crystal, and the scattering effect on macroscopic embodies a kind of volatility.

In terms of the mathematical description of the motion state of the scattering particle amount of a microscopic material particle after elastic wave scattering, we can use the schrodinger equation to express:

$$
i \hbar \frac{\partial \psi}{\partial t}=\hat{H} \psi
$$

Reflect the microscopic particles after wave scattering scattering particle motions changing with the time. Does not change over time time-independent schrodinger equation, the physical wave equation is represented as:

$$
\hat{H} \psi=E \psi
$$

In the same way, the strength of a material wave (the square of its amplitude) represents not the probability density of a particular place, but the number of scattered particles $N$.

$$
N \propto|\psi(r, t)|^{2}
$$

Therefore, I explained the origin of wave particle duality in microscopic matter particles. 


\section{4: A new interpretation of quantum entanglement}

Another fascinating aspect of quantum mechanics is the origin of quantum entanglement, an unsolved physics puzzle.

According to the above, the essence of quantum is the energy of a single wave. Because light is spherically symmetric potential $V(r) \psi$, so the light waves in office a little potential $d v$ in across the globe there will always be in the opposite direction of a symmetric potential point $d v^{\prime}$, we can turn any such a pair of symmetric potential points as a pair of entangled quantum. Therefore, quantum entanglement $\psi$ is a symmetric state of two potential points $d v$ and $d v^{\prime}$ on a light wave. The mathematical expression is:

$$
d v i d v^{\prime}
$$

When we measure the potential point on the light $d v$, will result in the light of spherically symmetric potential $V(r) \psi$ damage, leading to the ball of light waves in the symmetrical state of symmetrical potential disappear. (this is measuring led to the collapse of the wave $\psi$ function), causing potential points $d v^{\prime}$ off their symmetrical state, causing potential point $d v^{\prime}$ movement, this is the effect of quantum entanglement, which we explained the origin of the quantum entanglement.

You can see from the above analysis, quantum entanglement is a wave on two symmetric potential point of potential state of a form, this can be used to explain the quantum entanglement of all objects.

\section{5: Another possible interpretation for the cosmic red-shift}

By the spread of light is medium, so light is the medium of kinetic energy and potential energy, i.e., the Hamiltonian, the spread of the light waves through a medium will need to be overcome in the process of medium Hamiltonian, we define this quantity for Hamiltonian damping coefficient, thus, because the energy loss in the process of transmission of light, so the frequency of the light wave will reduce, satisfy the mathematical relationship is as follows:

$$
T=\frac{2 \pi}{\omega}=\frac{2 \pi}{\left(\omega_{0}^{2}-\beta^{2}\right)^{\frac{1}{2}}}
$$

Can be seen from the type, when light energy loss occurs in the process of transmission, the light frequency decreases, and spectrum redshift occurs, so that the red shift and damping coefficient of the Hamiltonian: proportional relationship, and because of the damping coefficient and the amount of dense, the light propagation proportional relation, thus, the red shift and proportional relationship:

$$
\lambda \propto \beta \propto r
$$

Therefore, it can be used to explain the red shift of the universe. If the red shift caused by the decrease of frequency per unit distance is expressed as a constant, then the relationship between the total red shift and the propagation distance is as follows:

$$
d_{\lambda}=k \times r
$$

As can be seen from the above, the further away the star is from us, the greater the energy loss of the star and the greater the red shift. This provides an alternative explanation for the spectral redshift of the universe.

Discussion: according to the above analysis, the nature of light, as a kind of medium wave will cause energy loss in the communication process, because of work, and loss of energy will be converted to heat energy, so, we can predict that our space generally have a heat temperature, but it just can be used to explain the source of the value of the existence of the cosmic microwave background radiation temperature.

\section{6: Another possible interpretation for the invariant principle of the speed of light}

A misunderstood the speed of light, the same principle: according to Galileo relativity principle, the mechanics principle in any inertial system are equivalent. So, for the speed of light, the light of the gravitational field medium relative stationary distribution, we in any of the gravity field measurement of the speed of light is relative to the gravitational field static medium for inertial reference frame measuring the speed of a universal value, we can define this phenomenon as follows: relative speed invariance principle. Due to waves are transmitted to earth from the sun, from a galaxy is a switch to another department, and there is no distance between two department of gravity, so the light switch between two gravity system satisfy the equation: 


$$
\left\{\begin{array} { l } 
{ x ^ { \prime } = x } \\
{ y ^ { \prime } = y } \\
{ z ^ { \prime } = z } \\
{ t ^ { \prime } = t }
\end{array} \text { or } \quad \left\{\begin{array}{l}
x=x^{\prime} \\
y=y^{\prime} \\
z=z^{\prime} \\
t=t^{\prime}
\end{array}\right.\right.
$$

The principle of relative velocity invariance is obtained by solving the first derivative of the first three equations in the above formula with respect to time:

$$
\left\{\begin{array}{l}
v_{x}^{\prime}=v_{x} \\
v_{y}^{\prime}=v_{y} \\
v_{z}^{\prime}=v_{z}
\end{array}\right.
$$

It can be predicted from the above expression that the sound velocity can also meet the principle of speed invariance by switching propagation in two inertial frames of reference. That is, we can do a plane and the velocity of the ground communication experiment, the people on the ground to measure the aircraft from the internal acoustic velocity is 340 $\mathrm{m} / \mathrm{s}$, is not a plane at a speed of $240 \mathrm{~m} / \mathrm{s}$ and the sound velocity of $340 \mathrm{~m} / \mathrm{s}$ after superposition of 580 $\mathrm{m} / \mathrm{s}$.

Reason: the sound waves in the process of spreading from inside the plane into the ground, there is a medium of inertial system switch, when the sound waves propagated to the aircraft skin, can cause the plane shell vibration, for the observer on the ground, the sound source is derived from the aircraft skin, and internal and not from the plane. This principle can be used to explain the invariant principle of the speed of light, indicating that when light waves switch from one inertial system to another, there is a secondary emission.

Then, the principle of relative speed of light invariance can be expressed as:

$$
\left\{\begin{array}{l}
c_{x}^{\prime}=c_{x} \\
c_{y}^{\prime}=c_{y} \\
c_{z}^{\prime}=c_{z}
\end{array}\right.
$$

Its vector form is:

$$
c^{\prime}=c
$$

It can be seen from the above that the speed of light is constant and does not satisfy the superposition principle. The reason is that when light waves switch from one gravitational system to another, there is a secondary emission of light waves. We usually measure the speed of light values that are light wave transmission medium for the reference value of the measured the speed of light, medium and light waves are always relative to the gravitational field, so, the speed of light in any speed measurement in the gravitational field values are the same. Thus, we say that the speed of light has nothing to do with the selection of reference frame, does not mean that the speed of light and the speed of light is the universe limit absolutely. Also is that we put forward another a feasibility interpretation about the speed of light, the same original way.

Discussion: can be seen from the above, the spread of light, medium is not absolute spatial distribution, but relative to the different gravitational field relative stationary distribution, spatial distribution of medium to and there is no absolute "ether" Michelson Morley experiment.

In addition, we all know that the speed of light is definitely a feasible explanation for the principle of constant speed of light, but it is not the only explanation. Because the level of cognition in the early 20th century was not enough to let us know the dynamic mechanism of light emission. Therefore, at that time, the origin of light waves could not be explained, and the speed of light could not be characterized. Therefore, we can only describe the nature of the speed of light propagation from the phenomenon, so Einstein proposed that the speed of light is an absolute conjecture (This was actually a very advanced point of view at the time). Of course, now it seems that this is not necessarily true. Because any speed is relative, isn't the speed of light not speed? Therefore, I have proposed another feasibility explanation about the principle of constant speed of light.

\section{Conclusion}

This paper aims to find a feasible interpretation of the origin of wave-particle duality of microscopic particles, so as to solve the pain points of quantum mechanical probability waves. Finally, the paper also unexpectedly discovered another way of explaining the feasibility of the principle of the redshift of the universe and the constant speed of light.

In the existing theory of quantum mechanics, we believe that particles of microscopic matter have 
wave-particle duality, and we cannot use one of them to fully interpret another property. In this paper, we have found a way to explain the feasibility of using one of the attributes to interpret another.

Specifically, I proved that the quantum is the energy of a single wave; At the same time, I also prove that quantum entanglement is a symmetry phenomenon of two potential points of a wave; The wave-particle duality of light is due to the particle nature of the energy discontinuity caused by the wave crest and valley. The wave-particle duality of other particles is a scattering effect. This means that I can use the volatility of light to interpret the particle nature of light; the particle nature of matter can be used to interpret the volatility of other microscopic particles. Since the essence of light is wave, the probability distribution of light wave energy cannot be discussed, and the distribution of energy flow density of light wave energy can only be discussed, thus eliminating the confusion of probability wave.

At the same time, we can see that the interpretation of Copenhagen is a feasible interpretation of the microscopic particle wave-particle duality based on the mathematical point of view, not the only interpretation. Therefore, this paper proposes another feasibility interpretation of the origin of the wave-particle duality of microscopic particles.

In addition, because the origin of light is determined in this paper, I also derived another feasibility interpretation of the principle of the redshift of the universe and the constant speed of light.

\section{Reference}

[1] Gutzwiller M C. Chaos in classical and quantum mechanics[J]. Physics Today, 1991, 44(11):94-96.

[2] Duarte F J. The origin of quantum entanglement experiments based on polarization measurements[J]. The European Physical Journal H, 2012, 37(2):311-318.

[3] Genovese M. Interpretations of Quantum Mechanics and the measurement problem[J]. Journal of Computational \& Theoretical Nanoscience, 2010, 3(3):249.

[4] Mittelstaedt P. The Interpretation of Quantum Mechanics and the Measurement Process[M]// The interpretation of quantum mechanics and the measurement process /. Cambridge University Press, 1998.

[5] Hollowood, Timothy J. The emergent Copenhagen interpretation of quantum mechanics $[\mathrm{J}]$. Journal of Physics A: Mathematical and Theoretical, 2014, 47(18):185301.

[6] Faye J. Copenhagen Interpretation of Quantum Mechanics[J]. Stanford Encyclopedia of Philosophy, 2008.

[7] Dewitt B S, Graham N. The Many Worlds Interpretation of Quantum Mechanics[M]. Princeton University Press, 1973.

[8] Adler S L. Why Decoherence has not Solved the Measurement Problem: A Response to P. W. Anderson[J]. Studies in History \& Philosophy of Modern Physics, 2001, 34(1):135-142.

[9] Tammaro E. Why Current Interpretations of Quantum Mechanics are Deficient[J]. Eprint Arxiv, 2014.

[10] Rowlands P. Quantum Uncertainty, Wave-Particle Duality and Fundamental Symmetries[M]// The Present Status of the Quantum Theory of Light. Springer Netherlands, 1997.

[11] Roy S. The Present Status of the Quantum Theory of Light[M]// The present status of the quantum theory of light: Kluwer Academic Pub. 1997.

[12] Bassi E B A, Dürr, Detlef, Weber T, et al. Quantum Mechanics: Are there Quantum Jumps? and On the Present Status of Quantum Mechanics[J]. 2006.

[13] Rashkovskiy S A. Is a rational explanation of wave-particle duality possible?[J]. 2013

[14] Jarosław Pykacz. Attempt at the Logical Explanation of the Wave-Particle Duality[J]. 1999.

[15] Lai M, Diels J C. Wave-particle duality of a photon in emission[J]. Journal of the Optical Society of America B, 1992, 9(12):2290-2294.

[16] Gatti A, Brambilla E, Lugiato L A. Entangled imaging and wave-particle duality: from the microscopic to the macroscopic realm[C]// European Quantum Electronics Conference. IEEE, 2003.

[17] Scully M O, Zubairy M S, Milonni P W. Quantum Optics[J]. Physics Today, 1998, 51(10):90-92. 